\title{
Efficacy of bone marrow mesenchymal stem cell transplantation in animal models of pulmonary fibrosis after exposure to bleomycin: A meta-analysis
}

\author{
ENGUO ZHANG ${ }^{1,2^{*}}$, YE YANG $^{1,2^{*}}$, JUAN ZHANG $^{1}$, GUOYONG DING $^{3}$, SHANGYA CHEN $^{1}$, CHENG PENG $^{1,4}$, \\ MARTIN F. LAVIN ${ }^{1,5}$, ABREY J. YEO ${ }^{1,5}$, ZHONGJUN DU $^{1}$ and HUA SHAO ${ }^{1}$ \\ ${ }^{1}$ Department of Toxicology, Shandong Academy of Occupational Health and Occupational Medicine, \\ Shandong Academy of Medical Sciences; ${ }^{2}$ School of Medicine and Life Sciences, \\ University of Jinan-Shandong Academy of Medical Sciences, Jinan, Shandong 250062; \\ ${ }^{3}$ School of Public Health, Taishan Medical University, Taian, Shandong 271016, P.R. China; \\ ${ }^{4}$ Queensland Alliance for Environmental Health Sciences, University of Queensland, Brisbane, Queensland 4102; \\ ${ }^{5}$ University of Queensland Centre for Clinical Research, University of Queensland, Brisbane, Queensland 4029, Australia
}

Received June 22, 2018; Accepted December 13, 2018

DOI: $10.3892 / \mathrm{etm} .2019 .7205$

\begin{abstract}
Previous studies have demonstrated that bone marrow mesenchymal stem cell (BMSC) transplantation is a promising treatment strategy for pulmonary fibrosis. Although encouraging results have been obtained using animal models of bleomycin (BLM)-induced pulmonary fibrosis, it is evident that transplantation of BMSCs at various time-points after BLM administration has produced different results in terms of treatment efficacy. To shed light on the potential utility of BMSCs for the treatment of lung disease, the present study performed a meta-analysis to estimate the efficacy of BMSCs in animal models of BLM-induced pulmonary fibrosis, and compare early transplantation (BMSCs injected on the same day after administration of BLM) with late transplantation (BMSCs injected on the 14th day after administration of BLM). Relevant studies were retrieved from the MEDLINE, PubMed, Chinese Knowledge Infrastructure and WanFang databases using a comprehensive search approach. A total of 6 studies
\end{abstract}

Correspondence to: Dr Zhongjun Du or Professor Hua Shao, Department of Toxicology, Shandong Academy of Occupational Health and Occupational Medicine, Shandong Academy of Medical Sciences, 18877 Jing-Shi-Road, Jinan, Shandong 250062, P.R. China E-mail: duzj1981@163.com

E-mail: chinashaohua5888@163.com

*Contributed equally

Abbreviations: BMSCs, bone marrow mesenchymal stem cells; BLM, bleomycin; IPF, idiopathic pulmonary fibrosis; MD, mean difference; CI, confidence interval; ATS, American Thoracic Society; REM, random effects model; FEM, fixed effects model

Key words: bone marrow mesenchymal stem cells, bleomycin, pulmonary fibrosis, treatment, systematic review involving 228 model rats were included. Meta-analysis indicated that early BMSC transplantation was able to prevent or reduce BLM-induced alveolitis and pulmonary fibrosis, while late BMSC transplantation was able to reduce alveolitis, but there was no significant evidence regarding improvement of pulmonary fibrosis. Although BMSC therapy was identified to be generally beneficial in rodent models of BLM-induced pulmonary fibrosis, the efficacy of early transplantation appears to be more satisfactory; overall, the efficacy of transplantation of BMSCs at the acute inflammatory phase was more effective compared with that at the chronic fibrosis stage. Of note, regarding alveolitis and pulmonary fibrosis scores after late transplantation of BMSCs, the sensitivity analysis revealed that the scores were less stable; thus, this result must be interpreted with caution. Furthermore, the quality and methodology of the included studies was comparatively low. Therefore, higher-quality and more rigorous studies are required to validate the results of the present meta-analysis in the future.

\section{Introduction}

Pulmonary fibrosis, particularly idiopathic pulmonary fibrosis (IPF), is a serious interstitial lung disease of unknown etiology, posing a major threat to human health. Due to the lack of effective drugs or treatments, the mortality rate associated with this condition is high. The median survival time of patients with IPF is estimated to be 3-5 years (1), which is worse compared with that of certain types of cancer (2). While the pathogenesis of pulmonary fibrosis is complex and has remained to be fully elucidated, the prevailing hypothesis is that repeated injury to the alveolar epithelium, together with a subsequent ineffective wound healing response and fibroblast activation, ultimately leads to pulmonary fibrosis (3-5).

The current understanding of the detailed pathogenesis of pulmonary fibrosis primarily relies on the use of animal models (6). An ideal animal model must resemble the basic 
characteristics of the human disease to allow for testing of specific hypotheses and their feasibility of constructing such a model in order to improve the understanding of the underlying mechanisms (7). To date, a number of animal species have been used as experimental models for studying pulmonary fibrosis, including mice, rats, $\operatorname{dog} s$, hamsters, guinea pigs and primates (8). When using experimental animals, multiple external interventions have been reported as capable of inducing pulmonary fibrosis, including bleomycin (BLM), silica or asbestos exposure, as well as cytokine overexpression, fluorescent isothiocyanate (FITC) and radiation (7,9-11). Among them, FITC is a chemical compound used to induce experimental pulmonary fibrosis. Fluorescein acts as a hapten and binds to airway proteins, thus acting as a depot for prolonged exposure to the injurious stimulus leading to fibrotic responses within 2-4 weeks that persist up to 24 weeks. Disadvantages of this model include absence of representative UIP characteristics and predominant inflammatory infiltrates that precede fibrosis (11). Although different animal models employing different interventions have their specific advantages and drawbacks, no single model has fully reproduced the histological pattern of interstitial pneumonia characteristic for human IPF to date. However, it is worth noting that animal models provide a key link between preclinical research and application of treatments in human clinical trials, and represent the foundation and a prerequisite for the development of treatments for human diseases. A recent consensus reached in an official American Thoracic Society workshop is that the use of the intratracheal BLM mouse model is the best-characterized animal model available for preclinical testing (12). BLM is a pro-fibrotic agent that causes DNA single- and double-strand breaks, with result in cell apoptosis and fibrosis. The advantage of intratracheal administration of BLM in mice is that it requires only a single instillation, which may better reproduce the development of fibrotic disease within a short period of time (7). For the treatment of IPF, a number of clinical trials have been completed based on preclinical studies in animal models, and two candidate drugs, namely pirfenidone and nintedanib, have emerged as potential treatments for IPF $(13,14)$. The two drugs were proven effective at slowing the progression of pulmonary fibrosis, although the treatment is not curative (3).

With the advancements in biological and medical technology, stem cell transplantation has been demonstrated to have a potential application in the treatment of various diseases. In this regard, bone marrow mesenchymal stem cells (BMSCs) have been indicated to be promising for inhibiting the onset and treating pulmonary fibrosis (15-18). BMSCs represent a class of non-hematopoietic stem cells in the bone marrow interstitium, with a high degree of self-replication and multidirectional differentiation potential. Of note, BMSCs have a strong immunosuppressive capacity with low immunoreactivity, rendering them safe as autografts or allografts (19). BMSCs have also been indicated to ameliorate BLM-induced pulmonary inflammation and fibrosis (20). A recent study using a similar model in aged mice reported that early intervention with allogeneic adipose-derived mesenchymal stromal cells attenuated lung and skin fibrosis and accelerated wound healing (21). In animal models, with regard to the frequency of dosing and the selection of treatment time-points, the majority of the experiments have examined the effects of a single intratracheal administration of BLM, and the selected time of treatment was usually within 1-7 days after BLM exposure (22-24). However, this type of approach examines the therapeutic effect on acute inflammation rather than on chronic fibrosis, limiting its application in human IPF.

Based on the abovementioned data, the present study aimed to examine the efficacy of BMSC intervention on BLM-induced pulmonary fibrosis models and compare the difference in efficacy between early and late intervention, focusing on pathology, including alveolitis and pulmonary fibrosis scores. By reviewing these preclinical data and providing a meta-analysis, the present study aimed to clarify certain issues, including the timing of treatment and its effectiveness on pulmonary fibrosis and, finally, the relevance of animal model studies in human IPF.

\section{Materials and methods}

Search strategy. The present study followed the Preferred Reporting Items for Systematic Reviews and Meta-Analysis guidelines (25). Studies examining the effect of BMSCs on animal models of pulmonary fibrosis published in Chinese or international biomedical journals between 2005 and 2017 were retrieved. All searches were performed in 2017 and the language was limited to English and Chinese. The search terms included 'bone marrow mesenchymal stem cells', 'pulmonary fibrosis', 'bleomycin' and 'animal model'. The search was performed in the PubMed, MEDLINE, Chinese Knowledge Infrastructure and WanFang databases to obtain comprehensive information.

Inclusion criteria and study selection. According to the specified inclusion and exclusion criteria, studies were first assessed by reading the title and abstract. The inclusion criteria were as follows: The study design focused on in vivo experiments of BLM-induced murine pulmonary fibrosis and the intervention was injection of BMSCs via the tail vein; the studies included a sufficient amount of samples (experimental and control groups); the exposure factor was intratracheal instillation of BLM; the intervention was injection of BMSCs via the tail vein; the gold standard for diagnosis and staging of diseases with increased fibrous tissue was histopathological analysis and the primary outcome was histopathological changes, including alveolar inflammation score and pulmonary fibrosis score; the inclusion of the design and research methods described above were similar and the data were complete. If the exposure factor was not BLM, if the intervention included cells other than BMSCs, or if the alveolitis or pulmonary fibrosis scores were not assessed, the study was excluded.

Data extraction and quality assessment. Two authors (E-GZ and YY) performed the search and selection of studies and disagreements were resolved by consultation. Data were entered into an Excel database by two authors (E-GZ and YY). The following data were extracted: The basic components of the selected studies (strains, sample size, intervention measures, number of injected cells, transplant time and outcome time) and the outcome indicators (alveolitis and pulmonary fibrosis scores). The methodological qualities were evaluated by two 
independent reviewers (E-GZ and YY) and differences were resolved by consulting a third reviewer (S-YC). The following items were assessed according to the standards recommended by the Cochrane Handbook 5.0 (26) and the SYRCLE animal experimental bias risk assessment tool (27). The biases assessed included randomization, blinding for researchers, blinding for outcome evaluators, selective reporting, concealed allocation and other biases.

Statistical analysis. Data were analyzed with Review Manager software, version 5.0 (Cochrane, London, UK). For continuous data, the mean difference (MD) and $95 \%$ confidence interval $(\mathrm{CI})$ were determined to assess the treatment effects. Data were pooled using the fixed-effects model (FEM), but the random-effects model (REM) was also considered to ensure the robustness of the model $(28,29)$. The percentage of variability across trials attributable to heterogeneity beyond chance was estimated using $\mathrm{I}^{2}$ statistics, which was deemed significant when $\mathrm{P}<0.05$ or $\mathrm{I}^{2}>50 \%$ (30). In case of significant heterogeneity $\left(\mathrm{I}^{2}>50 \%\right)$, the DerSimonian method and Laird REM were adopted as the pooling method. Subgroup analyses were performed according to different outcome times in the animal models after early BMSC transplantation. $\mathrm{P}<0.05$ was considered to indicate a statistically significant difference.

\section{Results}

Search results. After a comprehensive search, a total of 204 studies were retrieved, among which 6 were finally included according to the previously described inclusion criteria (31-36). A schematic of the screening process and selection of studies for inclusion in the meta-analysis is provided in Fig. 1.

Characteristics of included studies. The 6 experimental animal studies included in the present meta-analysis (31-36) comprised 228 model rats; of these, 131 were subjected to treatment and 97 were used as controls. All of the 6 studies were performed with a single tracheal injection of bleomycin at a dose of $5 \mathrm{mg} / \mathrm{kg}$. The studies mainly evaluated the histopathological changes in the lung after early and late transplantation of BMSCs. Early transplantation was defined as immediate transplantation of BMSCs after BLM administration. All of the 6 studies reported a score for alveolitis and pulmonary fibrosis after early transplantation of BMSCs. A total of 168 rats were included in the 6 studies. Late transplantation was defined as the transplantation of BMSCs on day 14 after administration of BLM to the rats, and a total of 3 studies, including 48 rats, evaluated the score of alveolitis and pulmonary fibrosis after late transplantation of BMSCs $(31,34,35)$. Detailed information on each study is presented in Table I. The results of the bias risk assessment for each of the included studies is provided in Table II. Only Huang et al (33) reported that sequence generation was grouped by the random number table method, the other studies did not describe a specific randomization method. No study described a concealed allocation, blinding for researchers and outcome evaluators.

Amelioration of alveolitis after early transplantation of BMSCs. FEM was used for meta-analysis, since the heterogeneity of the alveolitis score after early transplantation was $43 \%(<50 \%)$.

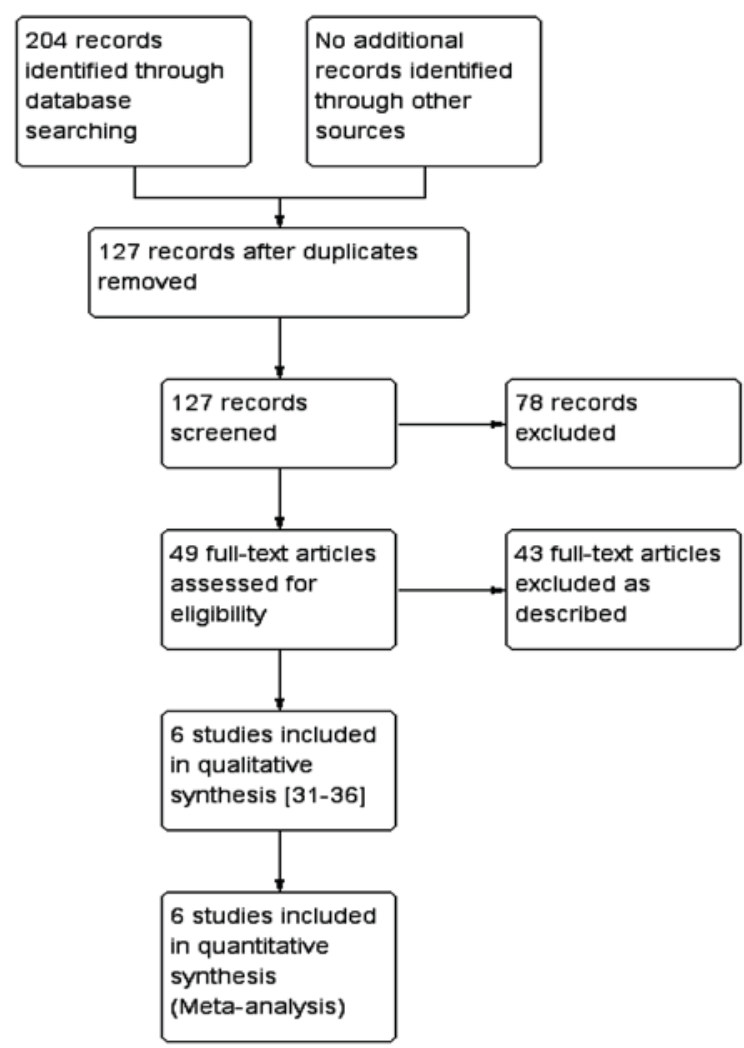

Figure 1. Flow diagram of the selection of study for inclusion in the present meta-analysis. As described refers to exposure factor not BLM, the intervention included cells other than BMSCs, or the alveolitis or pulmonary fibrosis scores were not assessed.

The meta-analysis results demonstrated that the alveolitis score in the early BMSC transplantation group was superior to that in the control group, with a statistically significant difference $[\mathrm{MD}=-0.68,95 \%$ CI $(-0.81,-0.56), \mathrm{P}<0.00001]$. The forest plot of the comparison is provided in Fig. 2. Further meta-analysis was performed for three subgroups defined by outcome time (day 7, 14 and 28). A total of 4 studies provided alveolitis score on days 7 and 14 after early transplantation in a total of 46 rats $(31,33,35,36)$. For day 7 , the REM was used for the meta-analysis, since heterogeneity existed among the 4 studies regarding the score on day $7\left(\mathrm{P}=0.07, \mathrm{I}^{2}=57 \%\right)$. For day 14 , the FEM was used for meta-analysis, since there was no heterogeneity among the 4 studies regarding the score for that day $\left(\mathrm{P}=0.42, \mathrm{I}^{2}=0 \%\right)$. The results demonstrated that the alveolitis score for the early transplantation group was significantly better compared with that in the control group on day $7[\mathrm{MD}=-0.84$, $95 \% \mathrm{CI}(-1.28,-0.40), \mathrm{P}=0.0002]$ and $14[\mathrm{MD}=-0.58,95 \% \mathrm{CI}$ $(-0.81,-0.35), \mathrm{P}<0.00001]$.

The alveolitis score on day 28 after early transplantation was reported by 5 studies including a total of 76 rats (31-35). The REM was used for meta-analysis, since heterogeneity was present among these 5 studies $\left(\mathrm{P}=0.08, \mathrm{I}^{2}=52 \%\right)$. The results demonstrated that the alveolitis score of the early transplantation group was better compared with that of the control group, with a statistically significant difference $[\mathrm{MD}=-0.86,95 \% \mathrm{CI}$ $(-1.20,-0.52), \mathrm{P}<0.00001]$.

Improvement of pulmonary fibrosis after early transplantation of BMSCs. REM was used for meta-analysis, 
Table I. Characteristics of the included studies.

\begin{tabular}{|c|c|c|c|c|c|c|c|c|}
\hline \multirow[b]{2}{*}{ Author, year } & \multirow{2}{*}{$\begin{array}{c}\text { Number of } \\
\text { samples }(\mathrm{T} / \mathrm{C})\end{array}$} & \multicolumn{2}{|c|}{ Intervention measures } & \multirow{2}{*}{$\begin{array}{l}\text { Number of } \\
\text { injected cells }\end{array}$} & \multirow{2}{*}{$\begin{array}{c}\text { Transplant } \\
\text { time after } \\
\text { modeling (days) }\end{array}$} & \multirow{2}{*}{$\begin{array}{c}\text { Outcome } \\
\text { time (days) }\end{array}$} & \multirow{2}{*}{$\begin{array}{l}\text { Outcome } \\
\text { indicator }\end{array}$} & \multirow[b]{2}{*}{ (Refs.) } \\
\hline & & $\mathrm{T}$ & $\mathrm{C}$ & & & & & \\
\hline Yang et al, 2016 & $30 / 15$ & BLM+BMSCs & BLM+saline & $0.2 \times 10^{7}$ & 1,14 & $7,14,28$ & $a, b$ & $(31)$ \\
\hline Cui et al, 2007 & $16 / 16$ & BLM+BMSCs & BLM+PBS & $2.5 \times 10^{6}$ & 1,7 & 28 & $a, b$ & (32) \\
\hline Huang et al, 2012 & $18 / 18$ & BLM+BMSCs & BLM+saline & $2.5 \times 10^{6}$ & 1 & $7,14,28$ & $a, b$ & (33) \\
\hline Wang et al, 2014 & $24 / 12$ & $\mathrm{BLM}+\mathrm{BMSCs}$ & BLM+saline & $1 \times 10^{6}$ & 1,14 & 28 & $a, b$ & (34) \\
\hline Wang et al, 2009 & $28 / 21$ & $\mathrm{BLM}+\mathrm{BMSCs}$ & None & $5 \mathrm{ml} / \mathrm{kg}$ & 1,14 & $7,14,28$ & $a, b$ & $(35)$ \\
\hline Zeng et al, 2016 & $15 / 15$ & BLM+BMSCs & BLM+saline & $5 \times 10^{5}$ & 1 & $7,14,21$ & $a, b$ & $(36)$ \\
\hline
\end{tabular}

In all studies, BMSCs were administered via tail vein injection. $\mathrm{T}$, treatment group; $\mathrm{C}$, control group; BMSCs, bone marrow mesenchymal stem cells; BLM, bleomycin; d, day; a, alveolitis; b, pulmonary fibrosis.

Table II. Bias risk assessment of the included studies.

\begin{tabular}{|c|c|c|c|c|c|c|c|}
\hline Author, year & Randomization & $\begin{array}{l}\text { Concealed } \\
\text { allocation }\end{array}$ & $\begin{array}{l}\text { Researcher } \\
\text { blinding }\end{array}$ & $\begin{array}{c}\text { Outcome } \\
\text { evaluator blinding }\end{array}$ & $\begin{array}{l}\text { Selective } \\
\text { reporting }\end{array}$ & Other biases & (Refs.) \\
\hline Yang et al, 2016 & Not described & Not clear & Not clear & Not clear & Not clear & Not clear & (31) \\
\hline Cui et al, 2007 & Not described & Not clear & Not clear & Not clear & Not clear & Not clear & (32) \\
\hline Huang et al, 2012 & $\begin{array}{l}\text { Random number } \\
\text { table method }\end{array}$ & Not clear & Not clear & Not clear & Not clear & Not clear & (33) \\
\hline Wang et al, 2014 & Not described & Not clear & Not clear & Not clear & Not clear & Not clear & (34) \\
\hline Wang et al, 2009 & Not described & Not clear & Not clear & Not clear & Not clear & Not clear & $(35)$ \\
\hline Zeng et al, 2016 & Not described & Not clear & Not clear & Not clear & Not clear & Not clear & (36) \\
\hline
\end{tabular}

since the heterogeneity of the pulmonary fibrosis score after early transplantation was $73 \%(>50 \%)$. The meta-analysis results demonstrated that the pulmonary fibrosis score was significantly improved in animals undergoing early BMSC transplantation compared with that in the control group [MD $=-0.73,95 \%$ CI $(-0.99,-0.48), \mathrm{P}<0.00001]$. The forest plot of the comparison is provided in Fig. 3. Further meta-analysis was performed for 3 subgroups by outcome time (day 7, 14 and 28).

A total of 4 studies reported a score for pulmonary fibrosis on days 7 and 14 after early transplantation in 46 rats $(31,33,35,36)$. The FEM was used for the meta-analysis for day 7 , since no heterogeneity was among these 4 studies regarding the score on day $7\left(\mathrm{P}=0.92, \mathrm{I}^{2}=0 \%\right)$. For day 14 , the REM was employed for meta-analysis, since heterogeneity was present among the 4 studies regarding the score on day $14\left(\mathrm{P}=0.03, \mathrm{I}^{2}=67 \%\right)$. The results demonstrated that the pulmonary fibrosis score in the early transplantation group was significantly different from that in the control group on day $7[\mathrm{MD}=-0.42,95 \% \mathrm{CI}(-0.62,-0.21), \mathrm{P}<0.0001]$ and day 14 $[\mathrm{MD}=-0.65,95 \% \mathrm{CI}(-1.18,-0.13), \mathrm{P}=0.02]$.

A total of 5 studies reported on the pulmonary fibrosis score on day 28 after early transplantation, in a total of 76 rats (31-35). The REM was used for meta-analysis, since heterogeneity existed among these 5 studies $(\mathrm{P}=0.002$, $\mathrm{I}^{2}=77 \%$ ). The results demonstrated that the pulmonary fibrosis score of the early transplantation group was better compared with that of the control group, with a statistically significant difference [MD $=-1.04,95 \%$ CI $(-1.45,-0.63), \mathrm{P}<0.00001]$.

Improvement of alveolitis following late transplantation of BMSCs. A total of 3 studies reported on the alveolitis score following late transplantation of BMSCs $(31,34,35)$. The FEM was used for meta-analysis, since no heterogeneity was present among the 3 studies regarding the alveolitis score on day 28 $\left(\mathrm{P}=0.82, \mathrm{I}^{2}=0 \%\right)$. The results demonstrated that the alveolitis score on day 28 in the late transplantation group was better compared with that in the control group, with a statistically significant difference $[\mathrm{MD}=-0.46,95 \%$ CI $(-0.76,-0.16)$, $\mathrm{P}=0.003$; Fig. 4].

Pulmonary fibrosis score after late transplantation of BMSCs revealed no evidence of improvement. A total of 3 studies reported on the pulmonary fibrosis score following late transplantation of BMSCs $(31,34,35)$. The REM was used for meta-analysis, since heterogeneity was present among these 3 studies for the score on day $28\left(\mathrm{P}=0.05, \mathrm{I}^{2}=66 \%\right)$. No significant difference was observed in the score for pulmonary fibrosis following transplantation of BMSCs on day 14 after administration of BLM $[\mathrm{MD}=-0.51,95 \%$ CI $(-1.03,0.00)$, $\mathrm{P}=0.05$; Fig. 5].

Sensitivity analysis. In order to assess the stability of the results of the present study, the included studies were sequentially 


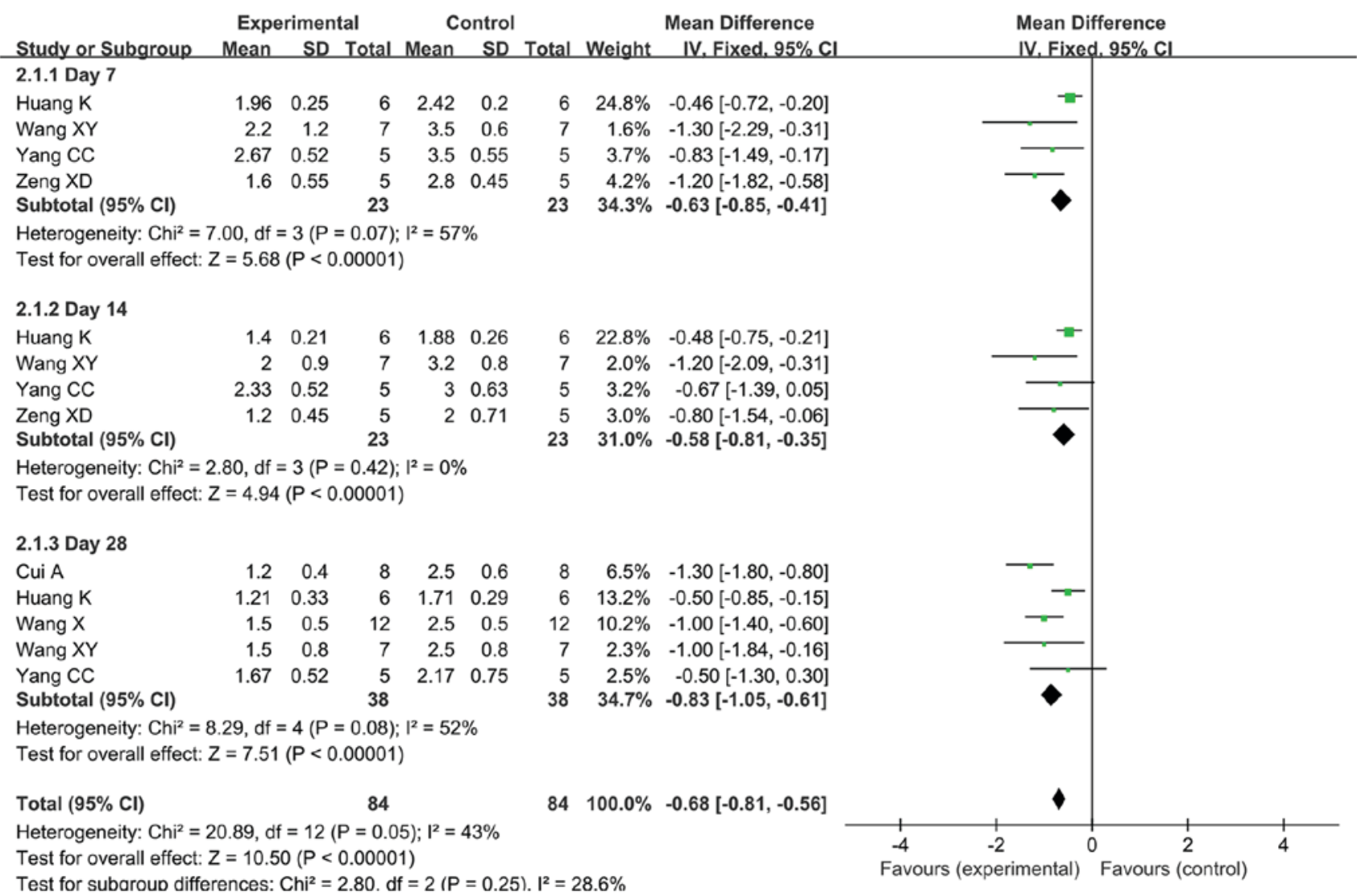

Figure 2. Forest plot for the comparison of bone marrow mesenchymal stem cell treatment vs. control (early transplantation) with alveolitis as the outcome. Horizontal lines describe the $95 \%$ CI; green squares represent the mean difference value and the size represents the weight of the study; black diamonds indicate the combined results of the effect size of each study. IV, inverse variance; df, degrees of freedom; SD, standard deviation; CI, confidence interval.

excluded, and the combined effect was re-estimated, and changes in the P-value and effect size were assessed. For the alveolitis scores, following late transplantation of BMSCs, upon exclusion of the data by Wang et al (34), which resulted in $\mathrm{MD}=-0.36,95 \%$ CI $(-0.99,0.27)$ and $\mathrm{P}=0.27$, the heterogeneity did not change. For the pulmonary fibrosis scores, following late transplantation of BMSCs, upon exclusion of the data from the study of Wang et al (34), which resulted in $\mathrm{MD}=-0.79,95 \% \mathrm{CI}(-1.22,-0.36)$ and $\mathrm{P}=0.0003$, the heterogeneity was reduced to $0 \%\left(\mathrm{I}^{2}=0 \%\right)$, indicating that the results of the late transplantation were sensitive and less stable. In order to explain this result, the study by Wang et al (34) was again reviewed. The number of BMSCs used in the study by Wang et al (34) $\left(1 \times 10^{6}\right)$ was lower than that of the amount of cells injected in the other studies (Table I), and this and may be one of the potential factors associated with bias. It may not be excluded that any further potential factors led to this result. Of note, this result must be interpreted with caution, and higher-quality and more rigorous studies are required to validate the authenticity and reliability of the result of late transplantation in the future.

Analysis of publication bias. The scores of alveolitis and pulmonary fibrosis were analyzed by funnel plots. The funnel plot for the alveolitis score after early transplantation of BMSCs was less symmetrical, suggesting the possible presence of publication bias (Fig. 6). The funnel plot for the pulmonary fibrosis score after early transplantation of BMSCs revealed that the two sides exhibited a degree of symmetry. Due to the limited number of studies involved, publication bias cannot be excluded (Fig. 7).

\section{Discussion}

In the present study, previously published experimental animal studies on BMSC transplantation for the treatment of BLM-induced pulmonary fibrosis we reviewed and subjected to a meta-analysis. The aim of the present study was to gain insight into the efficacy of this procedure and whether it may be employed as a potential approach for the treatment of human IPF. Specifically, histopathological changes, including alveolitis and pulmonary fibrosis scores, were examined in order to compare the efficacy of early transplantation of BMSCs with that of transplantation at a late stage on BLM-induced fibrosis in animal models. Early transplantation refers to BMSCs being injected on the same day after administration of BLM, with the rats sacrificed on days 7, 14 and 28, followed by assessment of alveolitis and pulmonary fibrosis. Late transplantation refers to the BMSCs injected on day 14 after administration of BLM, with the rats sacrificed on day 28 and the efficacy assessed in terms of alveolitis and pulmonary fibrosis. The criteria for scoring alveolitis and pulmonary fibrosis in the studies included were based on the method of Szapiel et al (37). In brief, alveolitis was evaluated using hematoxylin and eosin-stained sections and was graded as described therein, and fibrosis was determined using Masson's trichrome-stained sections and 


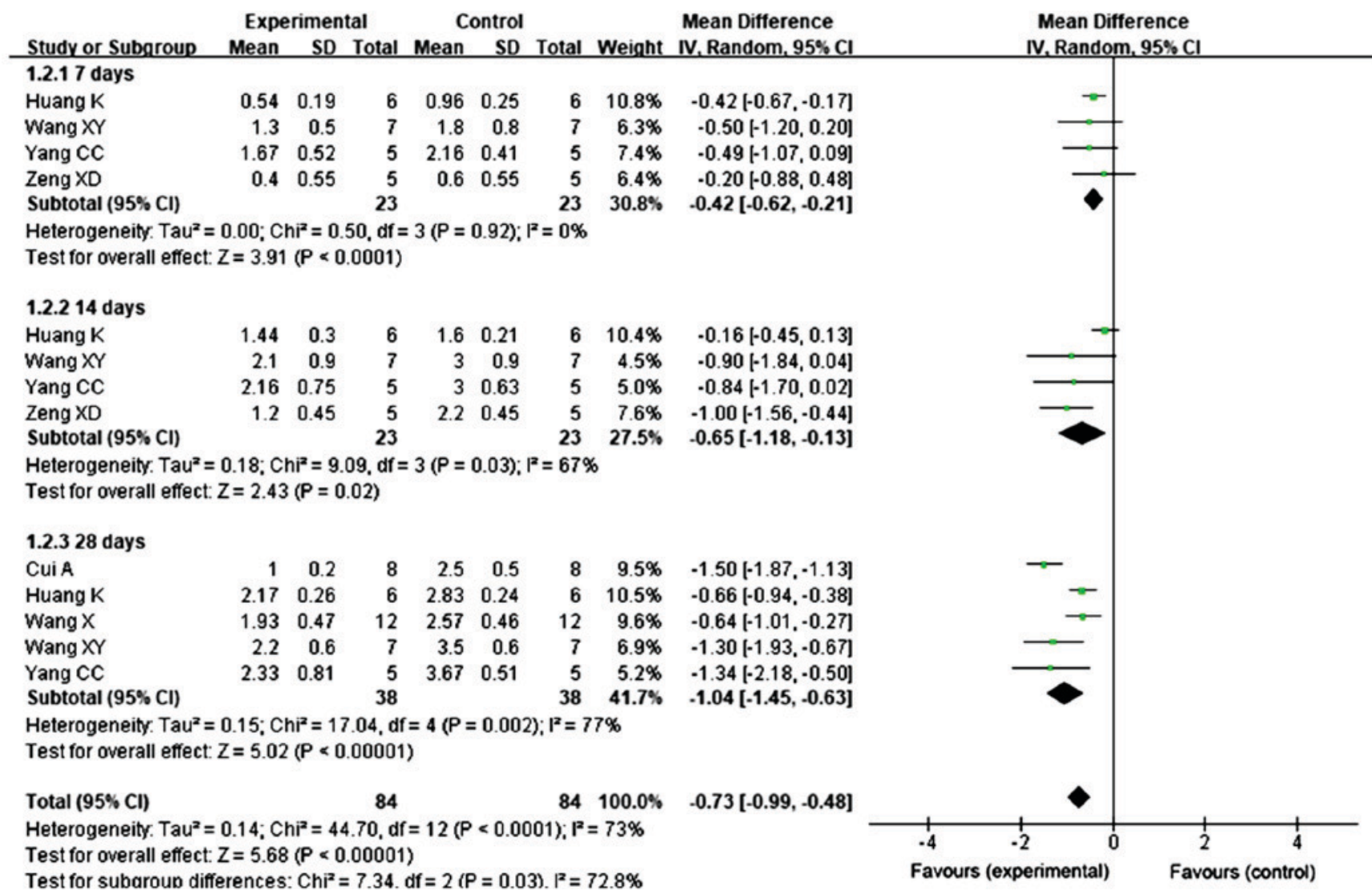

Figure 3. Forest plot for the comparison of bone marrow mesenchymal stem cell treatment vs. control (early transplantation) with pulmonary fibrosis as the outcome. Horizontal lines describe the 95\% CI; green squares represent the mean difference value and the size represents the weight of the study; black diamonds indicate the combined results of the effect size of each study. IV, inverse variance; df, degrees of freedom; SD, standard deviation; CI, confidence interval.

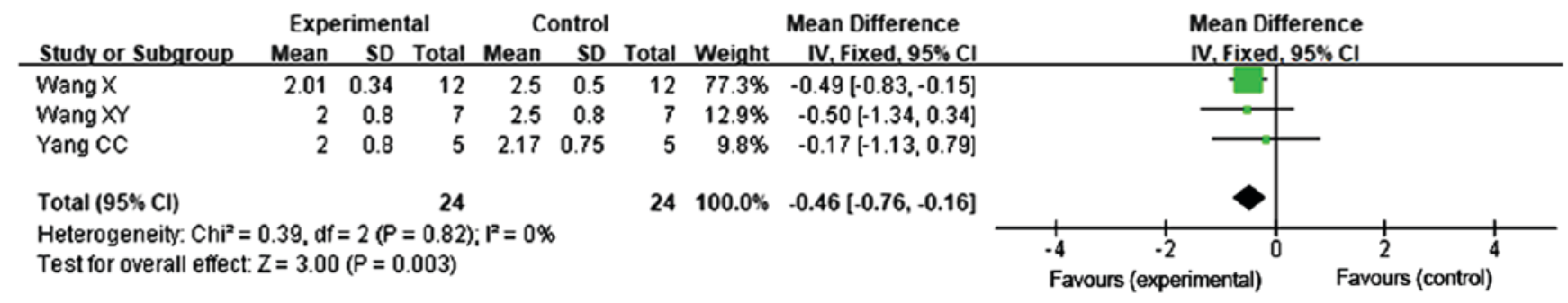

Figure 4. Forest plot for the comparison of bone marrow mesenchymal stem cell treatment vs. control (late transplantation) with alveolitis as the outcome. Horizontal lines describe the $95 \%$ CI; green squares represent the mean difference value and the size represents the weight of the study; black diamonds indicate the combined results of the effect size of each study.IV, inverse variance; df, degrees of freedom; SD, standard deviation; CI, confidence interval.

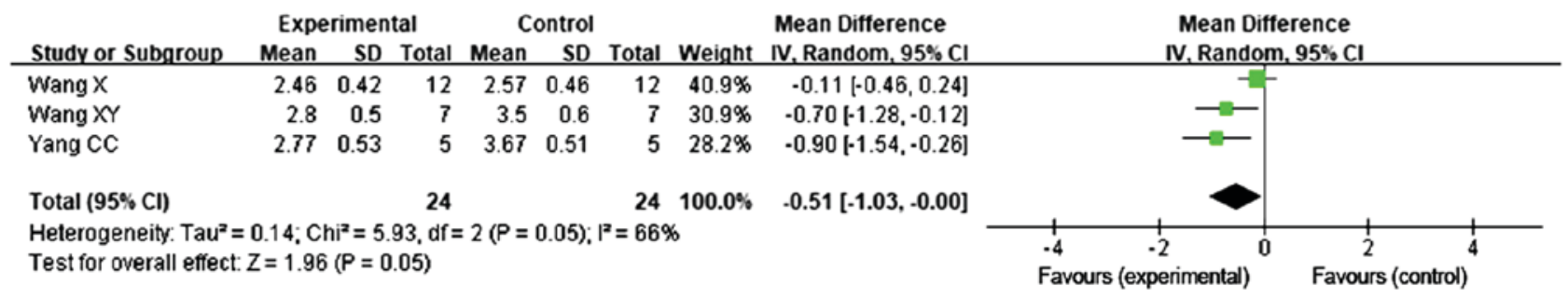

Figure 5. Forest plot for the comparison of bone marrow mesenchymal stem cell treatment vs. control (late transplantation) with pulmonary fibrosis as the outcome. Horizontal lines describe the $95 \% \mathrm{CI}$; green squares represent the mean difference value and the size represents the weight of the study; black diamonds indicate the combined results of the effect size of each study. IV, inverse variance; df, degrees of freedom; SD, standard deviation; CI, confidence interval.

again graded according to the criteria described. The criteria for alveolitis scoring in the study by Yang and $\mathrm{Wu}(31)$ were also as described above, and scoring of pulmonary fibrosis was a modification of that described by Szapiel et al (37), as 


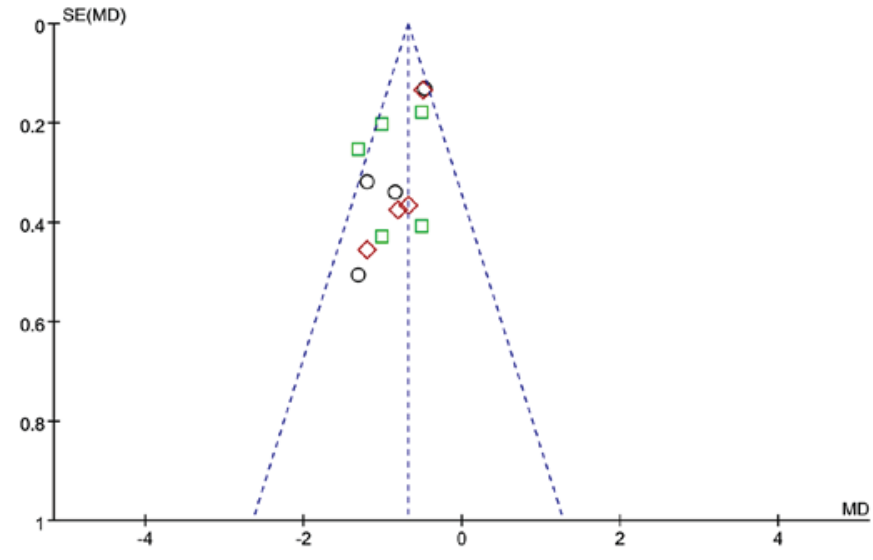

Oay $7 \stackrel{\text { Subgroups } 14 \square \text { Day } 28}{ }$

Figure 6. Funnel plot for the comparison of bone marrow mesenchymal stem cell treatment vs. control (early transplantation) with alveolitis as the outcome. SE, standard error; MD, mean difference.

described by Briggs et al (38), who reported subtle differences in the scores. Clearly, the approaches used to define these conditions were largely uniform. After review of the literature, a total of 6 studies were included in the present meta-analysis. The results of these studies demonstrated that alveolitis and pulmonary fibrosis scores were significantly improved at 7 , 14 and 28 days in the early transplantation group, indicating that early transplantation of BMSCs was able to ameliorate alveolitis and pulmonary fibrosis induced by BLM. In the late transplantation group, the results demonstrated that the alveolitis score, but not the pulmonary fibrosis score, were significantly different on day 28 after BMSC injection, suggesting that late transplantation of BMSCs may only lead to improvement of alveolitis. However, when the study by Wang et al (34) was excluded, since the number of injected cells or other potential factors were sources of bias in that study, the P-values of the scores of alveolitis and pulmonary fibrosis after late transplantation of BMSCs vs. the control were markedly changed, indicating that the sensitivity was higher and the stability of the result was lower. The results of the present meta-analysis demonstrated that late transplantation of BMSCs improves alveolitis, but there was no clear evidence regarding improvement of pulmonary fibrosis. However, this result must be interpreted with caution and higher-quality and more rigorous studies are required to validate the authenticity and reliability of these results in the future.

BLM is a chemotherapeutic antibiotic and has been identified as a pro-fibrotic agent (11). BLM-induced pulmonary fibrosis in animals has been considered to be the most commonly used experimental model. Of note, the severity of the course in BLM-induced pulmonary fibrosis in experimental animals depends on their age and sex. Previous studies reported that higher age and male sex may increase pulmonary inflammation and formation of fibrotic lesions, and that aged male C57BL/6 mice develop more severe BLM-induced pulmonary fibrosis compared with that of aged-matched female mice or younger mice of either sex (11). However, in general, pulmonary fibrosis induced by BLM usually develops rapidly through the following stages: i) Overproduction of reactive oxygen species in the first 3 days, leading to massive

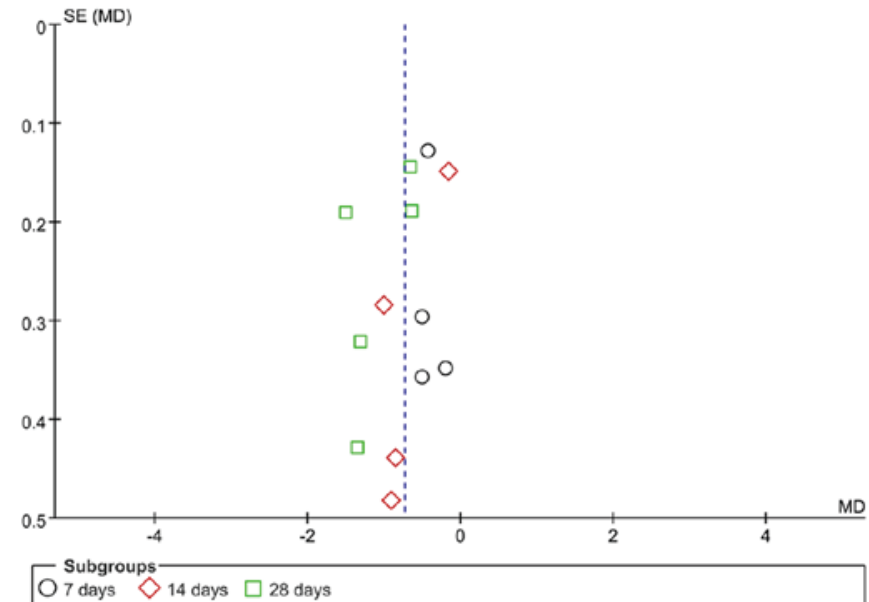

Figure 7. Funnel plot for the comparison of bone marrow mesenchymal stem cell treatment vs. control (early transplantation) with pulmonary fibrosis as the outcome. SE, standard error; MD, mean difference.

epithelial cell death; ii) formation of a large inflammatory cell infiltrate on days 3-9; iii) activation of fibroblasts with the deposition of a large amount of extracellular matrix and consequent occurrence of fibrosis on days 10-28 $(8,11,39,40)$. The first 7 days after exposure to BLM represent the acute inflammatory phase, after which time lung fibrosis gradually forms and peaks on the 14th day at the molecular and histological levels $(8,11)$. Thus, drug intervention during the first 1-7 days after exposure to BLM actually prevents inflammation, but not fibrosis per se. In the present meta-analysis, the appearance of inflammation occurred at the same time as the early transplantation of BMSCs, explaining for the higher effectiveness of this treatment in preventing fibrosis. Late transplantation of BMSCs was performed exactly on day 14 after BLM exposure, when fibrosis is at its peak. Therefore, treatment intervention at day 14 was efficacious in the treatment of acute fibrosis, and this result is of relevance in human IPF.

It has been previously reported that transplanting BMSCs at different time-points after treatment exhibited differential efficacy in treating pulmonary fibrosis in animal models. Early transplantation exerted a better effect compared with late transplantation (41). It is also noteworthy that, in previous studies with mice exposed to ionizing radiation and subsequently receiving late transplantation of BMSCs carrying green fluorescent protein, a transformation from macrophages to fibroblasts was observed, which defines the period of rapid development of fibrosis (42). Therefore, late transplantation of BMSCs may even accelerate pulmonary fibrosis. However, the present review provided evidence that BMSC therapy is beneficial in rodents with BLM-induced pulmonary fibrosis, and that the efficacy of early transplantation appears to be more satisfactory.

Of note, the present study has certain strengths and limitations. Through assessing the scores for alveolitis and pulmonary fibrosis, the present meta-analysis systematically evaluated the efficacy of early and late BMSC transplantation for the treatment of BLM-induced pulmonary fibrosis in rats. Data from animal studies provide important insight into the processes underlying pulmonary fibrosis, which may enhance the current understanding of the initiation, progression and development of human IPF. The results of the present study 
indicated that stem cell transplantation applied as an early intervention appears to be more effective. This is most likely due to the effect of early transplantation to inhibit inflammation in the early stage. This, in turn, suggests that early intervention by anti-inflammatory drugs may be effective in treating IPF in humans. Unfortunately, no effective biomarkers are currently available for early detection of human IPF, which makes early intervention difficult. While the animal models allow for the examination of the steps involved in the progression to fibrosis, it has not been fully elucidated whether similar changes occur during the development of human IPF.

Although late transplantation of BMSCs was able to reduce alveolitis, there was no clear evidence regarding the improvement of pulmonary fibrosis. However, for the alveolitis and pulmonary fibrosis scores following late transplantation of BMSCs were sensitive and less stable. Higher-quality and more rigorous studies are required to address the effectiveness of late intervention, since this strategy more closely resembles the treatment in humans.

Of note, the present study has certain limitations. For instance, the animal models reviewed only included rats. Previous studies reported that C57BL/6J mice are highly susceptible to lung injury following intratracheal BLM administration. Therefore, this strain may be an alternative model for investigating BLM-induced pulmonary fibrosis $(43,44)$. Another limitation was that the number of studies included was small and certain studies may have publication bias. Table II presents information on bias regarding allocation concealment, blinding and random assignments. For instance, only Huang et al (33) reported that rats were grouped by the random number table method, the other studies did not describe a specific random method. No study described concealed allocation, blinding for researchers and outcome evaluators (Table II). Although all studies used the tail vein injection route for cell transplantation, the transplant BMSC number was not equal (Table I). Of note, certain studies reported that exogenous transplanted BMSCs for silicosis exerted an antagonistic effect, with enhanced pathological pulmonary fibrotic changes due to excessive BMSC transplantation (17). The presence of a dose-effect association was evident. However, in the present study, it was not possible to determine whether the number of exogenous transplanted BMSCs affects BLM-induced pulmonary fibrosis. In addition, the SD was high in several studies, which may have affected the pooled data. Of note, the sensitivity analysis of the meta-analysis suggested that the results of late transplantation of BMSCs were sensitive and less stable. Therefore, it is required to interpret the results with caution. Higher-quality and more rigorous studies are required to validate the results of the present meta-analysis in the future.

In conclusion, the present study provided evidence that early transplantation of BMSCs improves the scores of alveolitis and pulmonary fibrosis induced by BLM in rat models. However, late transplantation of BMSCs was able to reduce alveolitis, but the majority of studies provided no evidence indicating improvement of pulmonary fibrosis. Although BMSC therapy appears to be beneficial in rodent models of BLM-induced pulmonary fibrosis, the efficacy of early transplantation appears to be more satisfactory, indicating that the efficacy of transplantation of BMSCs at the acute inflammatory phase is more effective compared with that at the chronic fibrosis stage. Of note, regarding the scores following late transplantation of BMSCs, the results were sensitive and less stable, and this result must therefore be interpreted with caution. More rigorous studies are required in the future to confirm the results of the present meta-analysis. In addition, most of the present data refer to the effect of early BMSC transplantation on the improvement of pulmonary fibrosis, while limited results are available for late transplantation. Therefore, it is recommended that future studies focus on transplantation of BMSCs at a more advanced stage and its long-term benefits.

\section{Acknowledgements}

Not applicable.

\section{Funding}

This study was supported by grants from the National Natural Science Foundation of China (grant no. 81602893), the Natural Science Foundation of Shandong Province (grant nos. ZR2015YL049 and ZR2018MH036), the Medical and Health Technology Development Plan Project of Shandong Province (grant no. 2016WS0540), the Key Research and Development Plan of Shandong Province (nos. 2017GSF18186 and 2018GSF118018) and the Innovation Project of Shandong Academy of Medical Science.

\section{Availability of data and materials}

The datasets generated and analyzed during the present study are available from the corresponding author upon reasonable request.

\section{Authors' contributions}

HS and ZD obtained funding and designed the study. EZ and YY performed the analysis and interpretation of the data, and wrote the manuscript. JZ, GD and SC performed the analysis and interpretation of data. AJY, CP and MFL provided technical support for the analysis and critical revision of the manuscript. All authors have read and approved the final version of the manuscript.

\section{Ethics approval and consent to participate}

Not applicable.

\section{Patient consent for publication}

Not applicable.

\section{Competing interests}

The authors declare that they have no competing interests.

\section{References}

1. Raghu G, Weycker D, Edelsberg J, Bradford WZ and Oster G: Incidence and prevalence of idiopathic pulmonary fibrosis. Am J Respir Crit Care Med 174: 810-816, 2006. 
2. Tzouvelekis A, Bonella F and Spagnolo P: Update on therapeutic management of idiopathic pulmonary fibrosis. Ther Clin Risk Manag 11: 359-370, 2015.

3. Ahluwalia N, Shea BS and Tager AM: New therapeutic targets in idiopathic pulmonary fibrosis. Aiming to rein in runaway wound-healing responses. Am J Respir Crit Care Med 190: 867-878, 2014.

4. Ryu JH, Moua T, Daniels CE, Hartman TE, Yi ES, Utz JP and Limper AH: Idiopathic pulmonary fibrosis: Evolving concepts. Mayo Clin Proc 89: 1130-1142, 2014

5. Tzouvelekis A and Kaminski N: Epigenetics in idiopathic pulmonary fibrosis. Biochem Cell Biol 93: 159-170, 2015.

6. Degryse AL and Lawson WE: Progress toward improving animal models for idiopathic pulmonary fibrosis. Am J Med Sci 341: 444-449, 2011.

7. Mouratis MA and Aidinis V: Modeling pulmonary fibrosis with bleomycin. Curr Opin Pulm Med 17: 355-361, 2011.

8. Moore BB and Hogaboam CM: Murine models of pulmonary fibrosis. Am J Physiol Lung Cell Mol Physiol 294: L152-L160, 2008.

9. Dong LH, Jiang YY, Liu YJ, Cui S, Xia CC, Qu C, Jiang X, Qu YQ, Chang PY and Liu F: The anti-fibrotic effects of mesenchymal stem cells on irradiated lungs via stimulating endogenous secretion of HGF and PGE2. Sci Rep 5: 8713, 2015.

10. Fireman E: A silica-induced pulmonary fibrosis model: Are we closer to 'real life' ? Int Arch Allergy Immunol 158: 211-212, 2012.

11. Tashiro J, Rubio GA, Limper AH, Williams K, Elliot SJ, Ninou I, Aidinis V, Tzouvelekis A and Glassberg MK: Exploring animal models that resemble idiopathic pulmonary fibrosis. Front Med (Lausanne) 4: 118, 2017.

12. Jenkins RG, Moore BB, Chambers RC, Eickelberg O, Königshoff M, Kolb M, Laurent GJ, Nanthakumar CB, Olman MA, Pardo A, et al: An official American thoracic society workshop report: Use of animal models for the preclinical assessment of potential therapies for pulmonary fibrosis. Am J Respir Cell Mol Biol 56: 667-679, 2017.

13. King TE Jr, Bradford WZ, Castro-Bernardini S, Fagan EA, Glaspole I, Glassberg MK, Gorina E, Hopkins PM, Kardatzke D, Lancaster L, et al: A phase 3 trial of pirfenidone in patients with idiopathic pulmonary fibrosis. N Engl J Med 370: 2083-2092, 2014

14. Richeldi L, du Bois RM, Raghu G, Azuma A, Brown KK, Costabel U, Cottin V, Flaherty KR, Hansell DM, Inoue Y, et al: Efficacy and safety of nintedanib in idiopathic pulmonary fibrosis. N Engl J Med 370: 2071-2082, 2014.

15. Li X, Wang Y, An G, Liang D, Zhu Z, Lian X, Niu P, Guo C and Tian L: Bone marrow mesenchymal stem cells attenuate silica-induced pulmonary fibrosis via paracrine mechanisms. Toxicol Lett 270: 96-107, 2017.

16. Ni S, Wang D, Qiu X, Pang L, Song Z and Guo K: Bone marrow mesenchymal stem cells protect against bleomycin-induced pulmonary fibrosis in rat by activating Nrf2 signaling. Int J Clin Exp Pathol 8: 7752-7761, 2015.

17. Zhao MM, Cui JZ, Cui Y, Li R, Tian YX, Song SX, Zhang J and Gao JL: Therapeutic effect of exogenous bone marrow-derived mesenchymal stem cell transplantation on silicosis via paracrine mechanisms in rats. Mol Med Rep 8: 741-746, 2013.

18. Zhou MI, Chen DL, Jiang T, Feng YM and Han XL: Effects of bone marrow-derived mesenchymal stem cells transfected with survivin on pulmonary fibrosis in mice. Exp Ther Med 10: 1857-1864, 2015

19. Abumaree M, Al Jumah M, Pace RA and Kalionis B: Immunosuppressive properties of mesenchymal stem cells. Stem Cell Rev 8: 375-392, 2012.

20. Yu SH, Liu LJ, Lv B, Che CL, Fan DP, Wang LF and Zhang YM: Inhibition of bleomycin-induced pulmonary fibrosis by bone marrow-derived mesenchymal stem cells might be mediated by decreasing MMP9, TIMP-1, INF- $\gamma$ and TGF- $\beta$. Cell Biochem Funct 33: 356-366, 2015

21. Rubio GA, Elliot SJ, Wikramanayake TC, Xia X, Pereira-Simon S, Thaller SR, Glinos GD, Jozic I, Hirt P, Pastar I, et al: Mesenchymal stromal cells prevent bleomycin-induced lung and skin fibrosis in aged mice and restore wound healing. J Cell Physiol 233: 5503-5512, 2018

22. Ortiz LA, Dutreil M, Fattman C, Pandey AC, Torres G, Go K and Phinney DG: Interleukin 1 receptor antagonist mediates the antiinflammatory and antifibrotic effect of mesenchymal stem cells during lung injury. Proc Natl Acad Sci USA 104: 11002-11007, 2007.

23. Ortiz LA, Gambelli F, McBride C, Gaupp D, Baddoo M, Kaminski N and Phinney DG: Mesenchymal stem cell engraftment in lung is enhanced in response to bleomycin exposure and ameliorates its fibrotic effects. Proc Natl Acad Sci USA 100: 8407-8411, 2003.
24. Rojas M, Xu J, Woods CR, Mora AL, Spears W, Roman J and Brigham KL: Bone marrow-derived mesenchymal stem cells in repair of the injured lung. Am J Respir Cell Mol Biol 33: 145-152, 2005.

25. Hutton B, Salanti G, Caldwell DM, Chaimani A, Schmid CH, Cameron C, Ioannidis JP, Straus S, Thorlund K, Jansen JP, et al: The PRISMA extension statement for reporting of systematic reviews incorporating network meta-analyses of health care interventions: Checklist and explanations. Ann Intern Med 162: 777-784, 2015.

26. Higgins JP and Sally G (eds): Cochrane Jandbook for Systematic Reviews of Interventions. A John Wiley \& Sons, Ltd., West Sussex, 2009.

27. Hooijmans CR, Rovers MM, de Vries RB, Leenaars M, Ritskes-Hoitinga M and Langendam MW: SYRCLE's risk of bias tool for animal studies. BMC Med Res Methodol 14: 43, 2014.

28. DerSimonian R and Kacker R: Random-effects model for meta-analysis of clinical trials: An update. Contemp Clin Trials 28: 105-114, 2007.

29. DerSimonian R and Laird N: Meta-analysis in clinical trials. Control Clin Trials 7: 177-188, 1986

30. Lau J, Ioannidis JP and Schmid CH: Quantitative synthesis in systematic reviews. Ann Intern Med 127: 820-826, 1997.

31. Yang CC and Wu XM: Inhibitory effect of bone mesenchymal stem cells on pulmonary fibrosis in rats. Chin J Int Med 14: 1091-1094, 2016 (In Chinese).

32. Cui A, Dai HP, Dai JW, Pang BS, Niu SJ, Lü YP and Wang C: Effects of bone marrow mesenchymal stem cells on bleomycin induced pulmonary fibrosis in rats. Zhonghua Jie $\mathrm{He} \mathrm{He} \mathrm{Hu} \mathrm{Xi} \mathrm{Za}$ Zhi 30: 677-682, 2007 (In Chinese).

33. Huang K, Wu XM, Wang XY, Kang XW, Xiao JL, Li ZG and Lu P: The effect of marrow mesenchymal stem cell transplantation on pulmonary fibrosis in rats. Zhonghua Jie $\mathrm{He} \mathrm{He} \mathrm{Hu} \mathrm{Xi}$ Za Zhi 35: 659-664, 2012 (In Chinese).

34. Wang X, Zeng Y and Peng H: Inhibitory effect of mesenchymal stem cells on pulmonary fibrosis in rats. Acta Med Univ Sci Technol Huazhong 3: 300-303, 2014

35. Wang XY, Wu XM, Huang K, Kang XW, Li B, Chen FH and Bai L: The treatment of experimental pulmonary fibrosis with bone mesenchymal stem cells transplantation. Chin J Rehab 3: 149-152, 2009 (In Chinese).

36. Zeng XD, Zhang $\mathrm{W}$ and Kang XH: Experimental study of mesenchymal stem cell transplantation in the treatment of bleomycin-induced pulmonary fibrosis in rats. J Gannan Med Uni 1: 20-23, 2016

37. Szapiel SV, Elson NA, Fulmer JD, Hunninghake GW and Crystal RG: Bleomycin-induced interstitial pulmonary disease in the nude, athymic mouse. Am Rev Respir Dis 120: 893-899, 1979.

38. Briggs BA, Bradley TM, Vernon P, Cooke NT, Drinkwater C, Gillett MK and Snashall PD: Measurement of lung tissue mass, thoracic blood and interstitial volumes by transmission/emission scanning using [99mTc]pertechnetate. Clin Sci (Lond) 73: 319-327, 1987.

39. Schrier DJ, Kunkel RG and Phan SH: The role of strain variation in murine bleomycin-induced pulmonary fibrosis. Am Rev Respir Dis 127: 63-66, 1983

40. Janick-Buckner D, Ranges GE and Hacker MP: Alteration of bronchoalveolar lavage cell populations following bleomycin treatment in mice. Toxicol Appl Pharmacol 100: 465-473, 1989.

41. Zhu HX, Gao JL, Zhao MM, Li R, Tian YX, Wang X, Zhang J and Cui JZ: Effect of bone marrow mesenchymal stem cell transplantation on silicosis fibrosis in different time windows in rats. Chin J Immun: 193-196,209, 2015.

42. Epperly MW, Guo H, Gretton JE and Greenberger JS: Bone marrow origin of myofibroblasts in irradiation pulmonary fibrosis. Am J Respir Cell Mol Biol 29: 213-224, 2003.

43. Hoyt DG and Lazo JS: Alterations in pulmonary mRNA encoding procollagens, fibronectin and transforming growth factor-beta precede bleomycin-induced pulmonary fibrosis in mice. J Pharmacol Exp Ther 246: 765-771, 1988.

44. Stefanov AN, Fox J, Depault F and Haston CK: Positional cloning reveals strain-dependent expression of Trim 16 to alter susceptibility to bleomycin-induced pulmonary fibrosis in mice. PLoS Genet 9: e1003203, 2013

This work is licensed under a Creative Commons Attribution-NonCommercial-NoDerivatives 4.0 International (CC BY-NC-ND 4.0) License. 